\title{
Effects of Modified Anodes on the Performance and Microbial Community of Microbial Fuel Cells Using Swine Wastewater
}

\author{
Hongjun $\mathrm{Ni}^{1,+}$, Kaixuan Wang ${ }^{1,2,+}$, Shuaishuai Lv ${ }^{1}$, Xingxing Wang ${ }^{1}$, Jiaqiao Zhang ${ }^{1,2}$, \\ Lu Zhuo ${ }^{1}$ and Fei Li $^{3, *}$ \\ 1 School of Mechanical Engineering, Nantong University, Nantong 226019, China; ni.hj@ntu.edu.cn (H.N.); \\ wang_kaixuan123@163.com (K.W.); lvshuaishuai@ntu.edu.cn (S.L.); wangxingxing.ntu@gmail.com (X.W.); \\ 1810310038@stmail.ntu.edu.cn (J.Z.); 1910310028@stmail.ntu.edu.cn (L.Z.) \\ 2 Graduate School of Advanced Technology and Science, University of Tokushima, Tokushima 770-8506, Japan \\ 3 Shanghai Key Laboratory for High Temperature Materials and Precision Forming, \\ Shanghai Jiao Tong University, Shanghai 200240, China \\ * Correspondence: sd532261601@163.com; Tel.: +86-1762-520-9162 \\ + The authors contribute equally.
}

Received: 11 July 2020; Accepted: 31 July 2020; Published: 2 August 2020

\begin{abstract}
Microbial fuel cells (MFCs) have emerged as a sustainable technology for wastewater treatment that has potential to recycle bioelectricity from livestock wastewater. The performance of MFCs is influenced by the synergistic effect of anode material with nearby microorganisms. In this study, three identical double-chambered MFCs with different anode carbon clothes using swine wastewater are established. The optimization mechanism of MFC performance is analyzed by anode characteristics, cell performance, and microbial community, respectively. The results show that the surface structure and properties of the anode carbon cloth can be obviously improved by the acid-heat-modified treatment. The community structure of anodic biofilm, which varied with different modification methods, was mainly dominated by Proteobacteria, Firmicutes, and Bacteroidetes. These findings demonstrate efficient and simple methods for improving the performance of MFCs based on swine wastewater and may help to explore the influence mechanism of different modified anodes on the exoelectrogens.
\end{abstract}

Keywords: microbial fuel cells; swine wastewater; modified anodes; anode characteristics; microbial community

\section{Introduction}

With the development of intensive livestock farms, the production efficiency and economic benefits of the livestock farms were improved significantly, but the relative concentration of livestock wastewater cannot be handled properly in many areas, which means that the livestock-farm wastewater has become or will be becoming a source of pollution equivalent to industrial wastewater or domestic sewage [1-3]. Many traditional wastewater treatment techniques are available to remove high concentrations of organic compounds from livestock wastewater, such as the returning farmland mode, the natural mode, and the factory mode [4,5]. The returning farmland mode and natural mode are poorly adapted and have the risk of secondary pollution. Although the factory model can meet the wastewater discharge standards, it entails high operational costs [6]. It is important to develop cost-effective treatments for the promising livestock wastewater with its high level of organics.

Microbial fuel cells (MFCs) have emerged as a sustainable technology for wastewater treatment that have potential in recycle bioelectricity from livestock wastewater and have great application 
prospects in livestock wastewater treatment, desalination, hydrogen production by electrolysis, and so on $[7,8]$. In a widely studied double-chambered MFC, some microorganisms suspended in the anode chamber degrade complex macromolecular organic compounds into small molecules, and exoelectrogens attached onto the anode oxidize simple organics and produce electrons and protons, simultaneously $[9,10]$. The generated electrons are transported to the anode surface in several ways and accepted by an electron acceptor in the cathode using an external circuit, while the protons are transferred to the cathode via the exchange membrane used to maintain the two chambers' (anode and cathode) relative independence [11,12]. The proton and electron arriving at the cathode have a three-phase reduction reaction with the electron acceptor in the cathode chamber, thus forming a complete current loop [13]. Applying MFCs to wastewater treatment can not only avoid many disadvantages of traditional techniques but also theoretically make the energy conversion efficiency of MFCs higher than that of biogas power generation without the limitation of the Carnot cycle, which accords with the purpose of social sustainable development [14-16]. However, the performance of MFCs is too poor to be applied in practice.

The performance of MFCs can be affected by the synergistic effect of the anode material with the surrounding microorganisms [17]. As the microorganism's attachment sites and electronic transit hub are in MFCs, the anode directly affects the electricity production and decontamination performance of the battery [18]. More and more researchers are trying to improve the anode materials reported or develop new anode materials and compare the effects of different anodes on the performance of MFCs for livestock wastewater treatment [19]. Although many studies have focused on the differences among modified anode materials characteristics and effects of various anodes on MFC performance, they did not analyze the effects of different anodes on the performance of MFCs using livestock wastewater as a substrate, especially swine wastewater, from the perspective of the biological community, and no scholars have determined the best modified anode of MFCs based on swine wastewater [20-22].

In this study, parallel-operated double-chambered MFCs equipped with untreated carbon cloth (CC), nitric acid-modified carbon cloth (N_CC), acid-heat-modified carbon cloth (N_H_CC), and ferric chloride-modified carbon cloth (F_CC) were constructed, using swine wastewater as a substrate, to compare the effects of different modification methods on the performance of MFCs. In particular, the effects of different modified anodes on the performance of MFCs based on swine wastewater were investigated from the view of microbial community structure, and the optimum method of CC anodic modification for MFCs with swine wastewater as a substrate was preliminarily determined. The experimental results have reference value for treating livestock wastewater, enhancing MFC performance and exploring the influence mechanism of different modified anodes on the production of microorganisms.

\section{Materials and Methods}

\subsection{Electrode Pretreatment}

First, all anode carbon cloths $(\varphi 16 \mathrm{~mm}, 0.21 \pm 0.01 \mathrm{~mm}$ thick, HCP120, Shanghai Hesen Electric Co., Ltd., Shanghai, China) were soaked in acetone for $3 \mathrm{~h}$ and then rinsed with the ultrapure water to neutral and dried at $60^{\circ} \mathrm{C}$ for spare, finally getting the CC. Then, one of the clean CCs was oxidized in $65 \%$ nitric acid at $50{ }^{\circ} \mathrm{C}$ for $6 \mathrm{~h}$ to obtain N_CC, and the other was soaked in $0.2 \mathrm{~mol} / \mathrm{L}$ ferric chloride solution for $4 \mathrm{~h}$ to obtain F_CC. The N_H_CC was prepared by heating N_CC at $450{ }^{\circ} \mathrm{C}$ for $2 \mathrm{~h}$, as shown in Figure 1. All modified carbon cloths were stored separately at $60{ }^{\circ} \mathrm{C}$ prior to use for further experiments. All cathodes were the same carbon cloth as anodes containing $0.35 \mathrm{mg} / \mathrm{cm}^{2} \mathrm{Pt}$ catalyst [23]. 


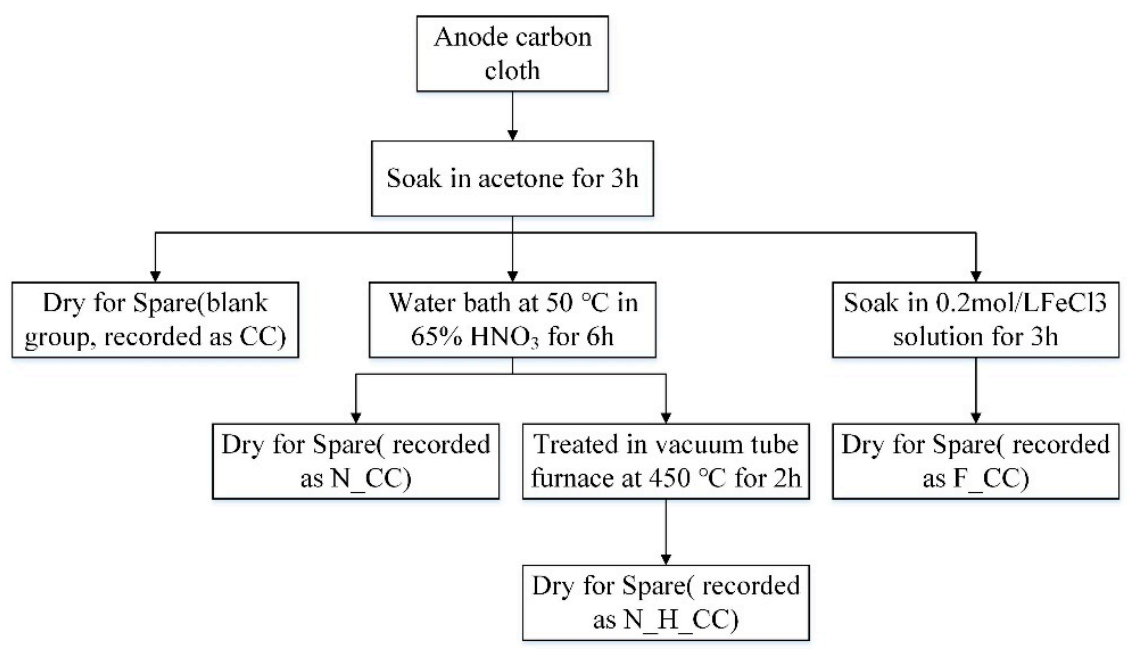

Figure 1. Anode modification roadmap.

\subsection{Construction and Operation}

Four identical reactors were fabricated by using transparent plexiglass to study the effects of different modification methods on the performance in identical MFCs, while keeping all other factors identical. The MFC reactor was separated into anode $(250 \mathrm{~mL})$ and cathode $(250 \mathrm{~mL})$ chambers by the Nafion 117 proton exchange membrane $(50 \mathrm{~mm} \times 50 \mathrm{~mm}$, Du Pont China Holding Co., Ltd., Beijing, China). The anode chamber of the MFCs was inoculated with a prefermentation supernatant of swine wastewater, a medium containing $1.0 \mathrm{~g} / \mathrm{L}$ sodium acetate and a $50 \mathrm{mM}$ phosphate buffer solution (PBS) containing $10 \mathrm{~mL} / \mathrm{L}$ trace minerals and $10 \mathrm{~mL} / \mathrm{L}$ vitamins [24]. The cathode chambers were inoculated with $0.1 \mathrm{~mol} / \mathrm{L}$ ferricyanide solution and a $50 \mathrm{mM}$ PBS. When the outputted voltage of the MFC reactor declined to below $50 \mathrm{mV}$ across an external loading resistance of $1000 \Omega$, a complete cycle was over. All reactors were operated in parallel-operated, fed-batch mode at $30{ }^{\circ} \mathrm{C}$.

\subsection{Analysis}

The morphology of modified CCs were examined using a scanning electron microscope (SEM) (S-3400N, Hitachi, Ltd., Tokyo, Japan). The functional groups carried by modified electrodes were examined with Vertex 70 FTIR (Clus scientific instruments (Shanghai) Co., Ltd., Shanghai, China). The surface wettability was measured with contact angle meter (DSA100, KRUSS GmbH, Hamburg, Germany). The external resistance voltages were collected using a data acquisition board (USB-6343, National Instruments Co., Ltd., Austin, TX, USA) with on-line monitoring and recording every $10 \mathrm{~min}$. When the voltage output became stable, data started to be recorded to obtain the polarization curve and power density curve by varying the resistance between the electrodes from $9999 \Omega-50 \Omega$. The polarization slope method was used to calculate the internal resistance (Rint) of MFC [25]. The power density of MFC were calculated according to the following equation [26]:

$$
\mathrm{P}_{\mathrm{An}}=\frac{\mathrm{U}^{2}}{\mathrm{R}_{\mathrm{ex}} \times \mathrm{S}_{\mathrm{An}}}
$$

where $P_{A n}$ is power density $\left(\mathrm{MW} / \mathrm{m}^{2}\right)$; $\mathrm{U}$ is operating voltage $(\mathrm{mV}) ; \mathrm{R}_{\mathrm{ex}}$ is external circuit resistance $(\Omega) ; S_{\text {An }}$ is anode electrode surface area $\left(\mathrm{m}^{2}\right)$.

After a week of stable operation of the MFCs, the sewage purified by MFCs was filtered with the drainage membrane $(0.45 \mu \mathrm{m}$, Jingteng Experimental Equipment Co., Ltd., Tianjin, China), and we examined chemical oxygen demand (COD) concentrations with a water-quality analyzer (DR3900, $\mathrm{HACH}$, Loveland, CO, USA). 
The DNA of MFC anode biofilm was picked up through a DNA isolation Kit (MoBio, Carlsbad, CA, USA). The V3-V4 regions of the $16 \mathrm{~S}$ rDNA gene were amplified and purified by the $16 \mathrm{~S}$ universal primers 338F (5'-ACTCCTACGGGAGGCAGCA-3') and 806R (5'-GGACTACHVGGGTWTCTAAT-3'). The high-throughput sequencing for polymerase chain reaction (PCR) amplification of barcoded $16 \mathrm{~S}$ rDNA gene was carried out on the Illumina Miseq platform at Majorbio Co., Ltd. (Shanghai, China). Based on $97 \%$ similarity, operational taxonomic units (OTUs) clustering analysis of qualified sequences were developed by the Sequence Read Archive (SRA, http://www.ncbi.nlm.nih.gov/Traces/sra) and were obtained by cut off, which were used for the analysis of microbial community diversity and the microbial community in different aspects.

\section{Results and Discussion}

\subsection{Characterizations of Anodes}

\subsubsection{SEM Analysis}

Different modification methods may affect the MFCs' performance by changing the anode characteristics. The SEM of carbon cloth anode treated by different methods is shown in Figure 2. SEM examination showed that the enlarged bulge structure makes the surface of the anode become rougher compared with the CC anode (Figure 2a), which may increase the specific surface area of the anode and be beneficial to the adhesion of microorganisms. The bulge structure of the N_H_CC (Figure 2c) anode was more uniform than N_CC (Figure 2b) or F_CC (Figure 2d), which may provide a better chemical reaction point for the anode and improve the battery performance. Particularly, the spatial distribution of carbon fibers on the F_CC anode appeared more compact than the other ones rarely reported before, which may reduce the anode specific surface area and weaken the microbial adhesion on the anode surface [27].
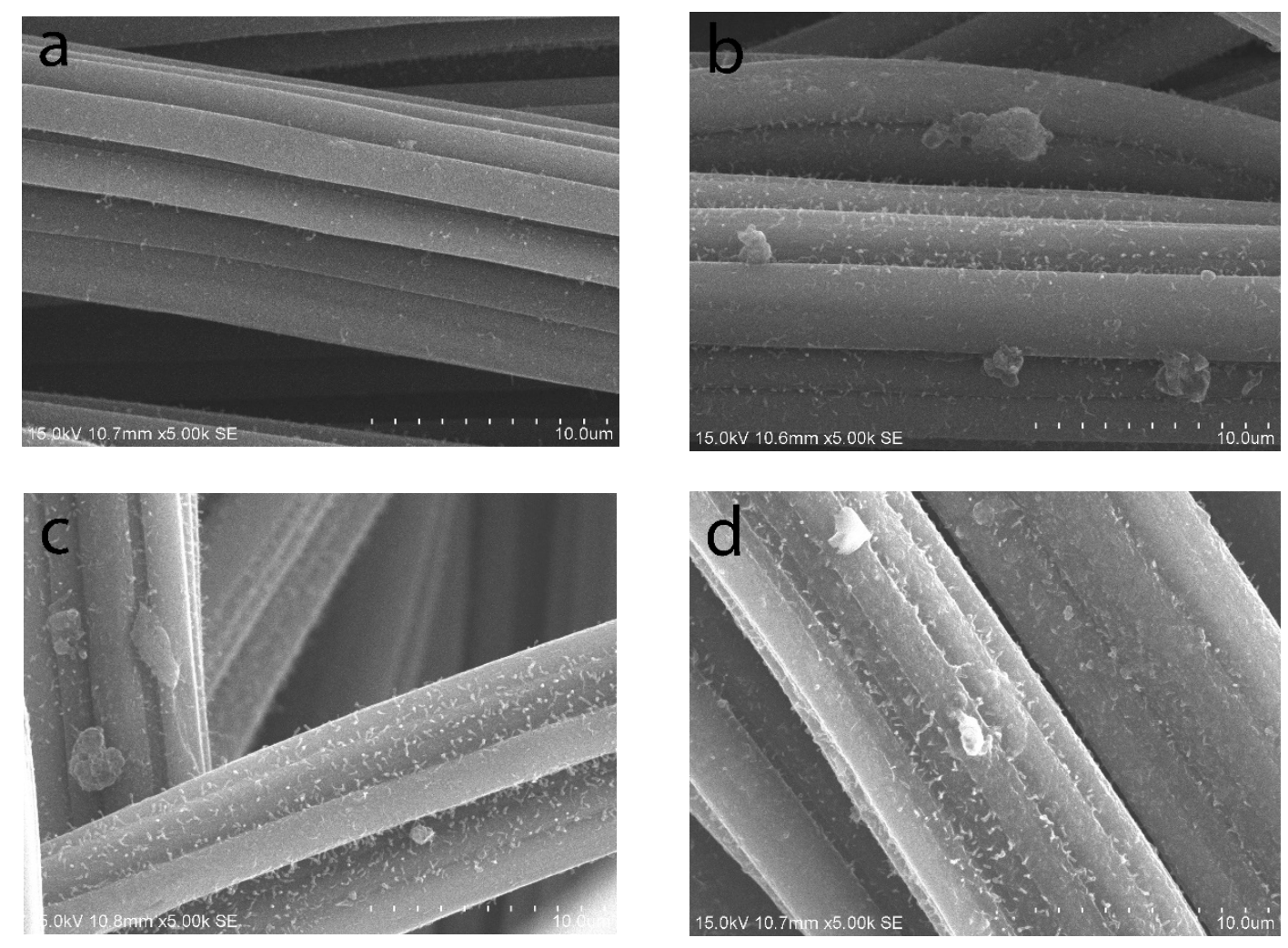

Figure 2. SEM images of a carbon cloth anode treated with different methods: (a) CC, (b) N_CC, (c) N_H_CC, and (d) F_CC. 


\subsubsection{Fourier Transform Infrared Spectroscopy (FT-IR) Analysis}

Modification treatment helped to increase the type of functional groups attached to the carbon cloth surface, which can be proved by the FT-IR spectra of the anode [28]. As shown in Figure 3, the infrared spectra of CC showed one peak at $2300-2400 \mathrm{~cm}^{-1}$, which was the asymmetric stretching vibration induced by a small amount of $\mathrm{CO}_{2}$ in the experiment. For N_CC, peaks at $3500-3800 \mathrm{~cm}^{-1}$ originate from the stretching vibration of $\mathrm{O}-\mathrm{H}$ band and $-\mathrm{NH}_{2}$ band, which was caused by the infrared radiation of $-\mathrm{COOH}$. The modified treatment resulted in more absorption peaks in the spectra of N_H_CC and F_CC. The stretching vibration of $\mathrm{C}=\mathrm{O}$ band at $1500-1700 \mathrm{~cm}^{-1}$ and $\mathrm{C}-\mathrm{O}$ band at $1000-1200 \mathrm{~cm}^{-1}$ were observed, respectively, in the infrared spectra, which may be caused by the carboxyl group (-COOH), while the absorption peak at $650-750 \mathrm{~cm}^{-1}$ was the outward flexural vibration of carboxyl $(\mathrm{O}-\mathrm{H})$. The results indicated that the hydrophilic groups were attached to the surface of the modified CC, such as hydroxyl, carboxyl, and amino groups, greatly improving the hydrophilicity of anode, which indirectly promoted the electron transfer rate benefiting from more microorganisms attachment to the anode and improved the overall performance of the MFCs.

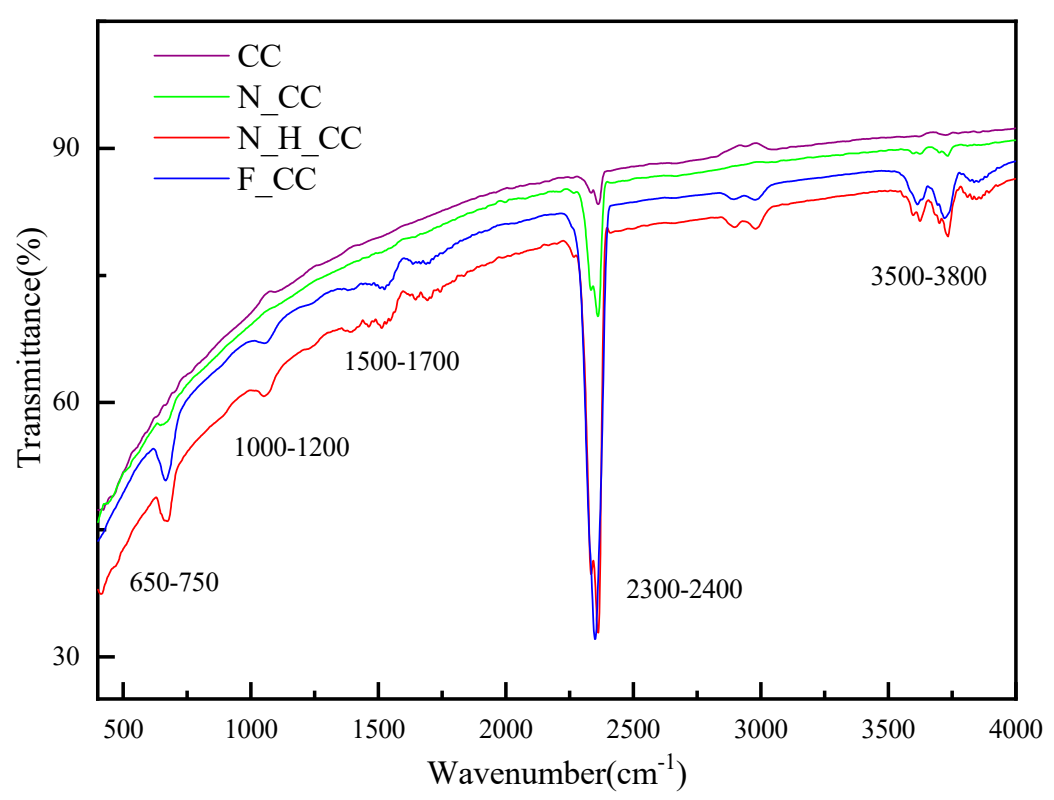

Figure 3. FT-IR spectra of the CC, N_CC, N_H_CC and F_CC.

\subsubsection{Contact Angle Analysis}

In order to further investigate the influence of different modification treatments on the characteristics of CC, especially the interaction between anolyte and anode, the wettability of different anodes was characterized by the contact angle [29]. As shown in Figure 4, the contact angle of CC $\left(132.2^{\circ}\right)$ was smaller than N_CC $\left(120.1^{\circ}\right)$ and F_CC $\left(122.9^{\circ}\right)$, indicating that the nitric acid and ferric chloride modification can enhance the wettability of carbon cloth relative to water, which was mainly due to the increased hydrophilic groups and bulge structure. The hydrophobicity of N_H_CC anode disappeared after modification, which may cause the partial change of the basic structure of carbon fiber and the decrease of the order of carbon fabric structure by modification, and the increased hydrophilic groups may be conducive to form hydrogen bonds with water. The results showed that the wettability of the anode was improved significantly after modification, which not only can enhance the compactness between the anode and the microorganism: by increasing the activity of microbial enzymes on the anode surface to advance the transfer between the microorganisms and anode, the potential of hydrophilic groups on a modified anode to act as electron-transfer carriers was also indirectly confirmed, and then the performance of the MFCs was improved. 

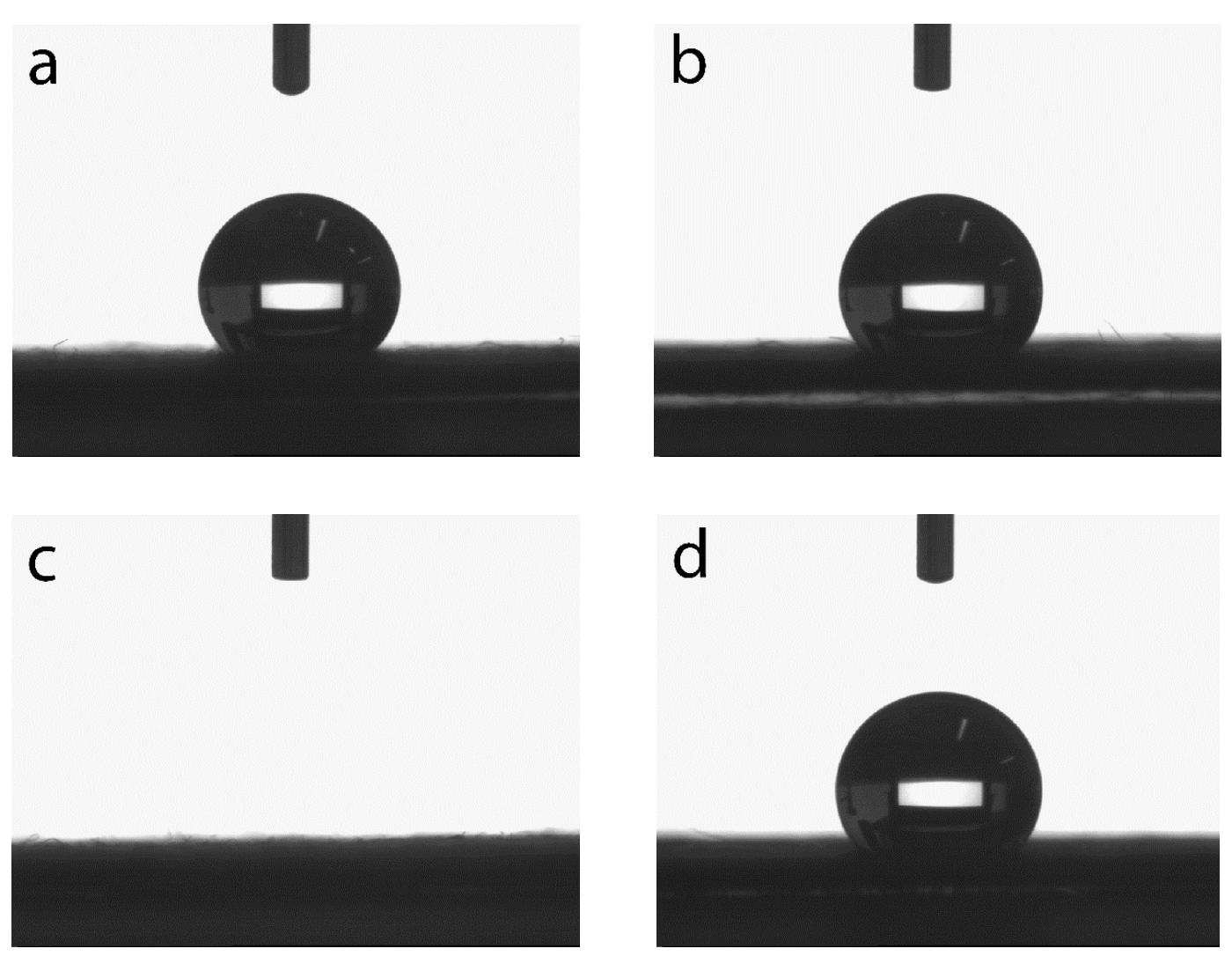

Figure 4. Contact angle diagram of the CC (a), N_CC (b), N_H_CC (c), and F_CC (d).

\subsection{MFC Performance}

Figure 5 shows the working voltage variation of four groups with different modified carbon cloth anodes. In the stable operation stage, the stable voltage of each group was $0.512 \mathrm{~V}(\mathrm{CC}), 0.550 \mathrm{~V}$ (N_CC), $0.634 \mathrm{~V}$ (N_H_CC), and $0.627 \mathrm{~V}$ (F_CC). It showed that the N_H_CC group performed better, and the voltage of N_H_CC was the highest, which was $23.82 \%$ higher than that of the CC. After the anode was modified, MFCs showed stronger power-generation performance, including shorter start-up period, longer stable operation stage, and higher stable voltage.

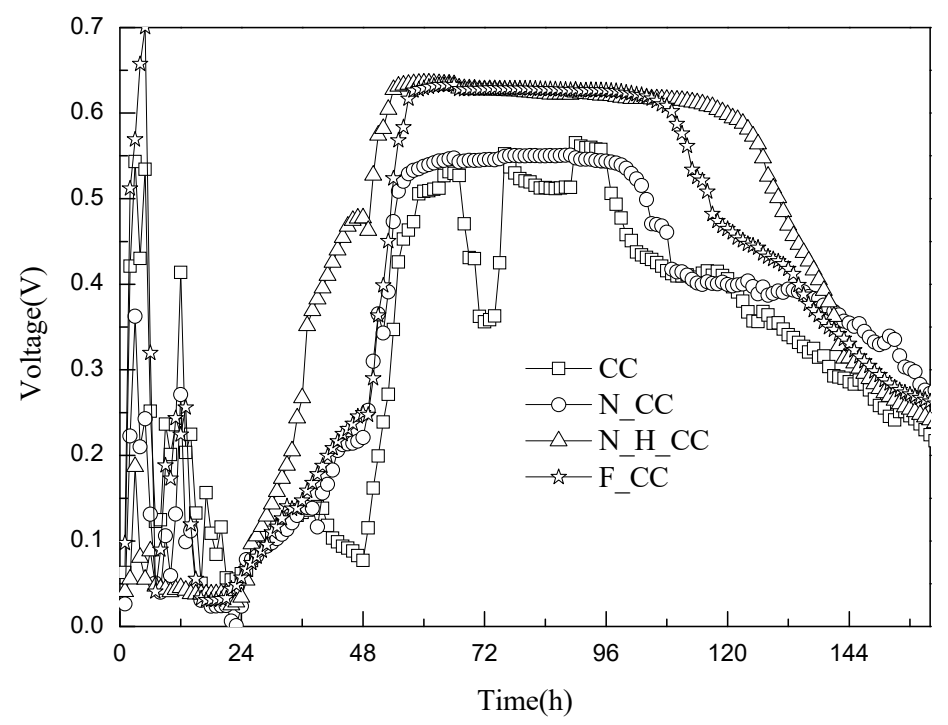

Figure 5. Working voltage of MFCs. 
The power densities and internal resistances of MFCs varied with the modification and could be obtained from the polarization curves [30]. Figure 6 showed that the maximum power densities for N_CC $\left(858.48 \mathrm{~mW} / \mathrm{m}^{2}\right)$ and N_H_CC $\left(883.62 \mathrm{~mW} / \mathrm{m}^{2}\right)$ were similar, followed by F_CC $\left(295.02 \mathrm{~mW} / \mathrm{m}^{2}\right)$ and CC $\left(196.68 \mathrm{~mW} / \mathrm{m}^{2}\right)$. The Rint was the external resistance value when the highest power density was reached. The minimum Rint for the N_H_CC reactor was $136 \Omega$, which was $0.27 \%, 34 \%$, and $95.77 \%$ of those for the CC, F_CC, and N_CC. The COD removal rates were 58.3\%, $71.2 \%, 80.1 \%$, and $61.9 \%$ for MFCs with CC, N_CC, N_H_CC, and F_CC, respectively, which was consistent with the power density trend. These results suggested that the improvement of the anode wettability and the increase of specific surface area promoted the electron transfer between the electrode and the microorganisms, which reduced the resistance of the MFCs and improved the ability to produce electricity or purify sewage, and it may be the main reason for the optimal performance of N_H_CC anode. Compared with N_CC, the average voltage of F_CC was high, but the power density was low, which may be because the introduction of more hydrophilic groups by the strong oxidation of $\mathrm{Fe}^{3+}$ reduced the internal resistance. However, agglomerated bundles of carbon fibers reduced the specific area of the F_CC anode and the adhesion of microorganisms, which resulted in the power density of the F_CC anode being lower than that of the N_CC anode and indirectly showed that the structure of the carbon anode and the adhesion of microorganisms have more influence on the performance of MFCs than the functional group or electron transfer on the electrode surface.

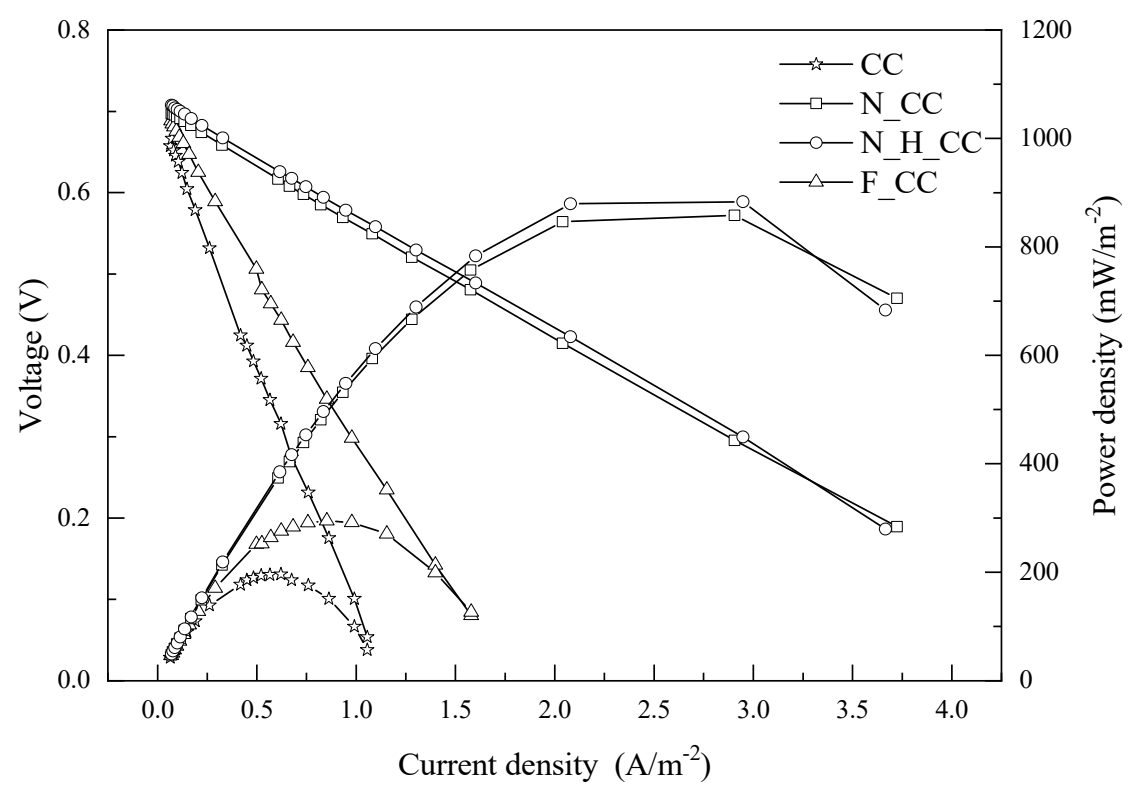

Figure 6. Power density and polarization curves of MFCs operated with CC, N_CC, N_H_CC, and F_CC.

\subsection{Microbial Community Diversity}

The Illumina sequencing analysis was adopted to generate a total of 139,591 raw sequences and obtained a total of 26,270, 31,232, 33,164, 34,536 high-quality reads from the anode biofilms of MFCs operated with CC, N_CC, N_H_CC, F_CC, respectively. A total of 509 (CC), 552 (N_CC), 425 (N_H_CC), 412 (F_CC) operational taxonomic units (OTUs) were determined at $97 \%$ nucleotide identify. The species richness and diversity of environmental communities were analyzed by calculating indexes.

As shown in Table 1, the observed Chao and Shannon estimators showed that the biofilm of N_CC had the highest richness and diversity, followed by CC, F_CC and N_H_CC. The Heip of F_CC was the highest among the four samples, implying that species evenness of F_CC was higher than CC, N_CC, and N_H_CC. The results showed that the space structure or hydrophilic groups attached to the carbon cloth after nitric acid modification can improve the microbial community diversity in the anode chamber, but it does not help much in the performance of MFCs. It is possible that more nonelectric 
microorganisms are attached to the anode, resulting in a decrease in the production of MFC with N_CC, and we should not neglect the fact that many dominant bacteria were degraded in the process of organic-matter consumption, which also proved that microbial community diversity of an MFC anode based on swine wastewater was not positively correlated with MFC performance. The F_CC and N_H_CC anode showed a more obvious selective effect on microorganisms, which caused a lower microbial community diversity than that of CC. The microbial community evenness of N_H_CC was lowest, indicating that the selection on the microbial community by the N_H_CC anode may develop better exoelectrogens or related cooperative communities to improve the power generation and decontamination performance of MFCs using swine wastewater. The values of coverage tend to be 1 , indicating that the sequencing data in the microbial diversity analysis was sufficient to reflect species diversity in the samples.

Table 1. Similarity based OTUs and species richness estimates.

\begin{tabular}{ccccccc}
\hline Sample & Reads & OTU & Chao & Heip & Shannon & Coverage \\
\hline CC & 26,270 & 509 & $\begin{array}{c}543 \\
(527,574)\end{array}$ & 0.1852 & $\begin{array}{c}4.56 \\
(4.54,4.58)\end{array}$ & 0.9977 \\
\hline N_CC & 31,232 & 552 & $\begin{array}{c}565 \\
(557,585)\end{array}$ & 0.1189 & $\begin{array}{c}4.83 \\
(4.81,4.85)\end{array}$ & 0.9989 \\
\hline N_H_CC & \multirow{2}{*}{33,164} & 425 & $\begin{array}{c}475 \\
(452,517)\end{array}$ & 0.0856 & $\begin{array}{c}3.63 \\
(3.61,3.65)\end{array}$ & 0.9980 \\
\hline F_CC & \multirow{2}{*}{34,536} & 412 & $\begin{array}{c}491 \\
(458,550)\end{array}$ & 0.2251 & $\begin{array}{c}3.91 \\
(3.9,3.93)\end{array}$ & 0.9975 \\
\hline
\end{tabular}

\subsection{Microbial Community Structure}

The predominant phyla in the anode biofilms of all MFCs belonged to Proteobacteria (78.71\%), Firmicutes (8.52\%), Bacteroidetes (7.44\%), Synergistetes (1.04\%), Verrucomicrobia $(0.95 \%)$, WS6 (0.86\%), Spirochaetes $(0.8 \%)$, and Saccharibacteria $(0.47 \%)$ in decreasing order. The highest relative abundance of Proteobacteria among the four samples were 68.7\% (CC), 92.65\% (N_CC), 82.83\% (N_H_CC), and 67.17\% (F_CC). Bacteroidetes can better adapt to the micro-ecological environment changes and help to improve the performance of MFCs. Synergistetes can degrade amino acids and pyruvate in an anaerobic environment and may play a role in degrading intermediate products of swine wastewater [31]. Verrucomicrobia, WS6, and Spirochaetes were commonly found in feces or soil but at low abundance in the reported anodic biofilm [32].

The relative abundance of the dominant populations showed that a response of microbial community to modification methods change had occurred, and the composition and relative abundance of microbial community at the phylum level were basically the same, but there were still differences, as shown in Figure 7. The relative abundance of dominant bacteria Proteobacteria was low in the microbial communities of CC or F_CC, which may be one of the main reasons leading to the poor performance of MFCs with CC or F_CC. The relative abundance of Proteobacteria in the N_CC anode was the highest, while its Firmicutes and Bacteroidetes relative abundance were the lowest, which affected the performance of MFCs to a certain extent and explained that the performance of N_CC was better than that of CC or F_CC. The optimal distribution of three dominant bacteria groups (Proteobacteria, Firmicutes, and Bacteroidetes) in the community structure of N_H_CC accounted for the optimum performance of MFCs with N_H_CC. The inexistence or low abundance of Synergistetes and Spirochaetes in the MFCs with N_CC and N_H_CC illustrated that they were of little benefit to the performance improvement of MFCs based on swine wastewater. Saccharibacteria existed only in N_H_CC and may effectively promote the fermentation or oxidation of swine wastewater. 


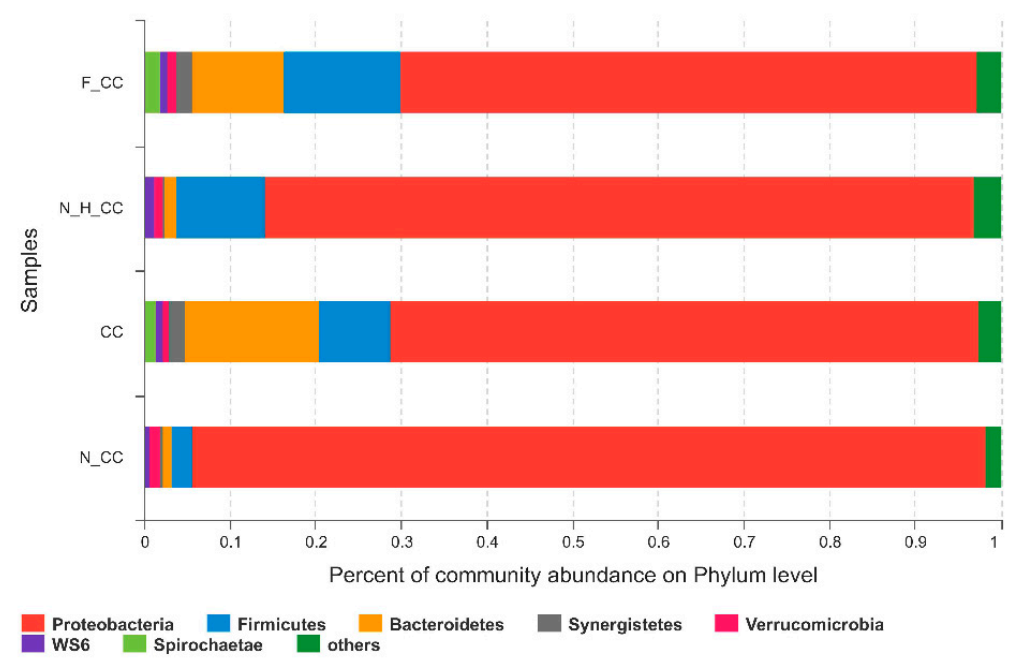

Figure 7. Relative ratio of the dominant bacterial at phylum level in CC, N_CC, N_H_CC, and F_CC.

Different samples of the hierarchical clustering tree on genus level is shown in Figure 8. The community composition of CC and F_CC was closer and so were N_CC and N_H_CC, which indicated that N_CC and N_H_CC had a more obvious selective effect on microorganisms than CC and F_CC.

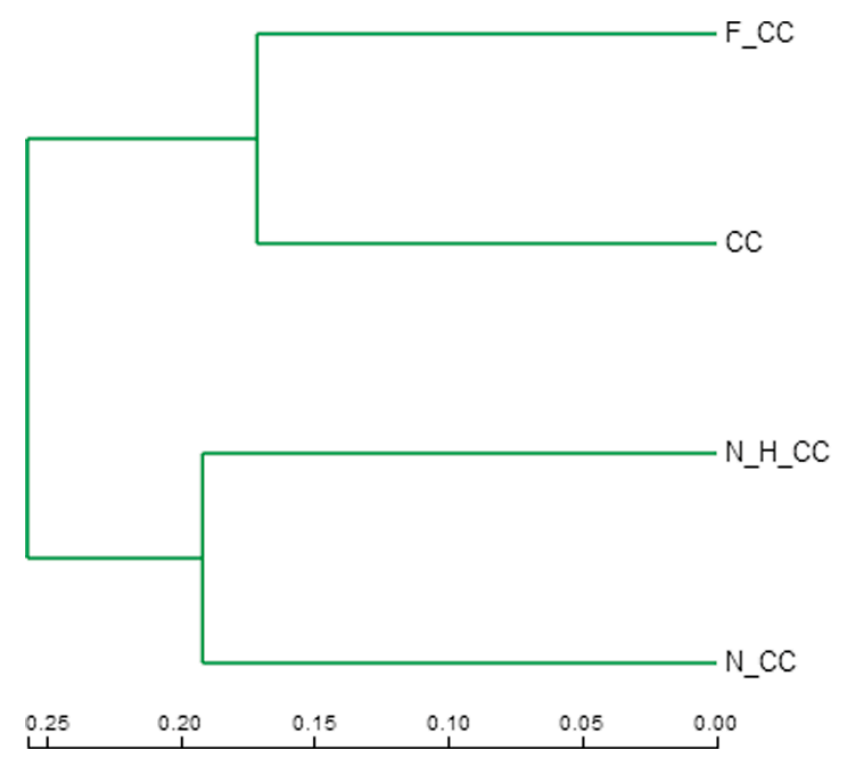

Figure 8. Hierarchical clustering tree on genus level for CC, N_CC, N_H_CC, and F_CC.

As shown in Table 2, the relative abundance $>1 \%$ species mainly distributed in Betaproteobacteria, Gammaproteobacteria, and Epsilonproteobacteria at the anode community. The dominant genus of relative abundance $>5 \%$ species in CC mainly included Methylomonas (10.52\%), Arcobacter $(9.15 \%)$, and Stenotrophomonas $(7.94 \%)$ in decreasing order, concentrated in Gammaproteobacteria and Epsilonproteobacteria. The dominant bacteria in F_CC were Thauera $(6.17 \%)$, Thermomonas $(6.73 \%)$, Comamonas (5.35\%), and the dominant genus in N_H_CC were Adevenella $(27.63 \%)$, Hydrogenophaga $(11.62 \%)$, and Comamonas $(6.91 \%)$. The number of dominant bacteria in N_CC was the largest, including Comamonas (11.96\%), Adevenella (11.04\%), Hydrogenophaga (10.34\%), Thermomonas $(8.36 \%)$ and Azoarcus (6.70\%). The dominant bacteria on the surface of the anode changed significantly after modification in which the relative abundance $>5 \%$ species mainly distributed in Betaproteobacteria and Gammaproteobacteria, and the content of dominant bacteria in N_CC and N_H_CC became less than 
before. The relative abundance $<1 \%$ species exceeding $1 / 3$ of the total sequence in CC and F_CC, which seriously limits the electricity production of microbial communities.

Table 2. Differences in relative abundance of the bacterial genera with CC, N_CC, N_H_CC, and F_CC.

\begin{tabular}{|c|c|c|c|c|c|}
\hline \multirow{2}{*}{ Phylum or Class } & \multirow{2}{*}{ Genus } & \multicolumn{4}{|c|}{ Abundance (\%) } \\
\hline & & $\mathrm{CC}$ & N_CC & N_H_CC & F_CC \\
\hline \multirow{7}{*}{ Alphaproteobacteria } & Sphingopyxis & 1.82 & 2.16 & 0.4 & 0.81 \\
\hline & Unclassified-f-Sphingomonadaceae & 0.37 & 2.18 & 3.6 & 0.9 \\
\hline & Novosphingobium & 0.89 & 1.63 & 2.34 & 0.98 \\
\hline & Unclassified-f-Caulobacteraceae & 0.99 & 1.94 & 0.24 & 0.68 \\
\hline & Phenylobacterium & 0.24 & 1.68 & 0.28 & 0.87 \\
\hline & Sphingobium & 0.09 & 1.60 & 0.74 & 0.63 \\
\hline & Brevundimonas & 0.95 & 1.14 & 0.58 & 0.74 \\
\hline \multirow{13}{*}{ Betaproteobacteria } & Comamonas & 4.89 & 11.96 & 6.91 & 5.35 \\
\hline & Aquabacterium & 3.44 & 1.26 & 1.75 & 1.42 \\
\hline & Adevenella & 2.73 & 11.04 & 27.63 & 6.26 \\
\hline & Hydrogenophaga & 2.59 & 10.34 & 11.62 & 4.2 \\
\hline & Castellaniella & 2.05 & 1.69 & 0.28 & 2.56 \\
\hline & Methylophilus & 1.09 & 0 & 0 & 0.13 \\
\hline & Unclassified-f-Comamonadaceae & 1.46 & 2.27 & 1.22 & 1.58 \\
\hline & Thauera & 0.95 & 7.76 & 3.98 & 6.17 \\
\hline & Azoarcus & 0.6 & 6.70 & 2.96 & 2.09 \\
\hline & Azovibrio & 0.29 & 0.24 & 1.41 & 0.12 \\
\hline & Norank-f-Alcaligenaceae & 0.17 & 3.34 & 1.08 & 0.97 \\
\hline & Simplicispira & 0.18 & 0.23 & 1.06 & 0.24 \\
\hline & Unclassified-f-Alcaligenaceae & 0.02 & 1.39 & 0 & 0.23 \\
\hline \multirow{5}{*}{ Gammaproteobacteria } & Methylomonas & 10.52 & 1.51 & 0 & 1.87 \\
\hline & Stenotrophomonas & 7.94 & 4.24 & 3.52 & 4.76 \\
\hline & Thermomonas & 4.57 & 8.36 & 1.78 & 6.73 \\
\hline & Acinetobacter & 1.17 & 0.26 & 0 & 0.3 \\
\hline & Pseudomonas & 0.5 & 0.22 & 0.16 & 1.69 \\
\hline \multirow{2}{*}{ Deltaproteobacteria } & Desulfomicrobium & 2.01 & 0.48 & 3.38 & 3.42 \\
\hline & Desulfobulbus & 0.44 & 1.10 & 1.9 & 0.63 \\
\hline Epsilonproteobacteria & Arcobacter & 9.15 & 0.64 & 0.53 & 4.3 \\
\hline \multirow{3}{*}{ Bacteroidetes } & vadinBC27-wastewater-sludge-group & 2.31 & 0 & 0.44 & 2.13 \\
\hline & Norank-c-Bacteroidetes-vadinHA17 & 1.5 & 0.12 & 0.1 & 1.75 \\
\hline & Flavobacterium & 1.44 & 0.12 & 0 & 0.99 \\
\hline \multirow{3}{*}{ Firmicutes } & Clostridium-sensu-stricto-1 & 1.14 & 0.48 & 3.76 & 2.45 \\
\hline & Erysipelothrix & 0.5 & 0.27 & 0.75 & 1.15 \\
\hline & Terrisporobacter & 0.55 & 0.39 & 1.9 & 1.1 \\
\hline Synergistetes & Norank-f-Synergistaceae & 1.56 & 0.18 & 0.23 & 1.56 \\
\hline$P-W S 6$ & Norank-p-WS6 & 0.92 & 0.56 & 1.16 & 0.83 \\
\hline others & & 27.98 & 10.31 & 12.06 & 27.41 \\
\hline
\end{tabular}

The four samples had the same dominated species at genus level, such as Comamonas, Hydrogenophaga, Stenotrophomonas, and Thermomonas. Comamonas and Thermomonas have been reported to be beneficial for improving MFC performance, and similar results have been obtained in this study, which were more densely distributed in MFCs with N_CC and N_H_CC positively correlated with cell performance. Hydrogenophaga and Stenotrophomonas were efficient bacteria reported for sewage treatment, and their enrichment was beneficial to the stability of microbial communities [33].

A small amount of Methylomonas was beneficial to the production of electricity cooperated with exoelectrogens, which was the only highly abundant genus of $\mathrm{CC}$, but too much of it may consume too many organics and limit the rest of exoelectrogens [34]. After modification, the abundance of 
Methylomonas on the anode surface decreased obviously, which indicated that the selection by modified anode on the biological community was beneficial for improving the performance of MFCs. The highest relative abundance species in N_H_CC was Advenella (27.6\%), which was widely distributed in swine wastewater and not proven to be effective for MFC. Advenella was demonstrated to enhance the degradation of substrate by sludge system, which may be one of the main reasons for the best decontamination capacity of N_H_CC. Azovibrio and Simplicispira has been shown to be better adapted to low temperature environments both of which belonged to N_H_CC peculiarly. Ferric chloride modification enabled Thauera to become the dominant genus in the F_CC, which, due to the strong ability of Thauera to reduce $\mathrm{Fe}^{3+}$, effectively promoted electron transfer to improve the performance of the MFCs.

From the analysis above, the effects of different modifications on microbial community structure on the carbon cloth anode was obvious. Although the electricity generation by many dominant genera in swine wastewater remains to be further confirmed, there is no doubt that the performance of MFC with N_H_CC using swine wastewater was the best among the four samples in this study.

\section{Conclusions}

Three different methods were used to modify the anode carbon cloth to explore the influence of anode modification on the power generation and decontamination performance of MFCs. From the perspective of the microbial community, the mechanism of different modification methods on the performance of MFCs based on swine wastewater was explored. The results showed that anode modification clearly influenced electricity generation and microbial community structure of MFCs. The space structure and properties of the carbon cloth anode can be improved obviously by acid thermal modification with which MFCs can produce the maximum power density $\left(883.62 \mathrm{~mW} / \mathrm{m}^{2}\right)$ and COD removal rate $(80.1 \%)$. Compared with the untreated carbon cloth, the power density was increased by $350 \%$. The microbial community structure and function analysis revealed that the selection by modified anode on the biological community was beneficial to improve the performance of MFCs. The dominant bacteria in MFCs were mainly distributed in Proteobacteria (Alphaproteobacteria, Betaproteobacteria, Gammaproteobacteria, Deltaproteobacteria, Epsilonproteobacteria), Firmicutes, and Bacteroidetes. Acid-heat modification was the best method for improving the MFC anode carbon cloth based on swine wastewater. Swine wastewater treated by MFCs can effectively solve the problem of environmental pollution and provide reference and future usage for improving the performance of MFCs using livestock wastewater. It also provides a new way for the development of renewable energy in practice and further research.

\section{Patents}

The authors of this paper have carried out research on the microbial fuel cell for many years. Four China invention patents related to this paper have been granted, the patent numbers are CN106784952B, CN106876760B, CN106848361B, and CN108075162B. In addition, four China invention patents have been published: the patent numbers are CN110713899A, CN110669644A, CN109081424A and CN109081450A.

Author Contributions: Conceptualization, H.N.; data curation, S.L., X.W., and F.L.; formal analysis, H.N. and K.W.; funding acquisition, F.L.; investigation, S.L.; methodology, K.W. and L.Z.; project administration, F.L.; resources, X.W.; software, K.W. and J.Z.; supervision, H.N., L.Z., and F.L.; validation, X.W. and L.Z.; visualization, S.L. and J.Z.; writing—original draft, K.W.; writing—review \& editing, H.N. All authors have read and agreed to the published version of the manuscript.

Funding: This work was supported by Priority Academic Program Development of Jiangsu Higher Education Institutions, grant number PAPD, and Nantong Applied Research Project, grant numbers JC2018115 and JC2019129.

Conflicts of Interest: The authors declare no conflict of interest. 


\section{References}

1. Corbella, C.; Hartl, M.; Fernandez-Gatell, M. MFC-based biosensor for domestic wastewater COD assessment in constructed wetlands. Sci. Total Environ. 2019, 660, 218-226. [CrossRef]

2. Mei, X.; Xing, D.; Yang, Y. Adaptation of microbial community of the anode biofilm in microbial fuel cells to temperature. Bioelectrochemistry 2017, 117, 29-33. [CrossRef]

3. Ni, H.J.; Zhuo, L.; Wang, X.X. Research progress of anode material for microbial fuel cell. Chin. J. Power Sources 2019, 43, 528-531.

4. Ni, H.J.; Wang, K.X.; Lv, S.S. Effects of Concentration Variations on the Performance and Microbial Community in Microbial Fuel Cell Using Swine Wastewater. Energies 2020, 13, 2231. [CrossRef]

5. Ulusoy, I.; Dimoglo, A. Electricity generation in microbial fuel cell systems with Thiobacillus ferrooxidans as the cathode microorganism. Int. J. Hydrogen Energy 2018, 43, 1171-1178. [CrossRef]

6. Pan, Y.; Zhu, T.; He, Z. Energy advantage of anode electrode rotation over anolyte recirculation for operating a tubular microbial fuel cell. Electrochem. Commun. 2019, 106, 106529. [CrossRef]

7. Neethu, B.; Pradhan, H.; Sarkar, P. Application of ion exchange membranes in enhancing algal production alongside desalination of saline water in microbial fuel cell. MRS Adv. 2019, 4, 1077-1085. [CrossRef]

8. Santoro, C.; Arbizzani, C.; Erable, B. Microbial fuel cells: From fundamentals to applications. Rev. J. Power Sources 2017, 356, 225-244. [CrossRef]

9. Nandy, A.; Sharma, M.; Venkatesan, S.V. Comparative Evaluation of Coated and Non-Coated Carbon Electrodes in a Microbial Fuel Cell for Treatment of Municipal Sludge. Energies 2019, 12, 1034. [CrossRef]

10. Zhu, J.P.; Wang, J.; Zhang, T.P. Phylogenetic Diversity of Bacterial and Archaeal Communities in Anode biofilm of Sediment Microbial Fuel Cells. Acta Sci. Circumstantiae 2016, 36, 4017-4024.

11. Yang, Y.; Choi, C.; Xie, G. Electron transfer interpretation of the biofilm-coated anode of a microbial fuel cell and the cathode modification effects on its power. Bioelectrochemistry 2019, 127, 94-103. [CrossRef] [PubMed]

12. Long, X.Z.; Wang, H.; Wang, C.Q. Enhancement of azo dye degradation and power generation in a photoelectrocatalytic microbial fuel cell by simple cathodic reduction on titania nanotube arrays electrode. J. Power Sources 2019, 415, 145-153. [CrossRef]

13. Hernandez, L.A.; Riveros, G.; Gonzalez, D.M. PEDOT/graphene/nickel-nanoparticles composites as electrodes for microbial fuel cells. J. Mater. Sci. 2019, 30, 12001-12011. [CrossRef]

14. Zhu, Y.; Zhu, Y.Y.; Wang, X.X. Research Progress of Active Components of the Eletrocatalysts for Direct Enthanol Fuel Cell. Nantong Univ. 2017, 16, 58-63.

15. Wang, G.C.; Yu, M.S.; Xie, K.W. Graphene modified polyacrylonitrile fiber as high-performance cathode for marine sediment microbial fuel cells. J. Power Sources 2019, 438, 227002. [CrossRef]

16. Liu, L.; Chou, T.Y.; Lee, C.Y. Performance of freshwater sediment microbial fuel cells: Consistency. Int. J. Hydrogen Energy 2016, 41, 4504-4508. [CrossRef]

17. Xie, G.; Choi, C. High performance Microbial Fuel Cell Using Metal Ion Complexes as Electron Acceptors. Bull. Korean Chem. Soc. 2020, 41, 348-357. [CrossRef]

18. Ma, J.; Ni, H.J.; Su, D.Y. Bioelectricity generation from pig farm wastewater in microbial fuel cell using carbon brush as electrode. Int. J. Hydrogen Energy 2016, 41, 16191-16195. [CrossRef]

19. Liu, D.; Chang, Q.; Gao, Y. High performance of microbial fuel cell afforded by metallic tungsten carbide decorated carbon cloth anode. Electrochim. Acta 2019, 330, 135243. [CrossRef]

20. Rossi, R.; Evans, P.J.; Logan, B.E. Impact of flow recirculation and anode dimensions on performance of a large scale microbial fuel cell. J. Power Sources 2019, 412, 294-300. [CrossRef]

21. Schilirò, T.; Tommasi, T.; Armato, C. The study of electrochemically active planktonic microbes in microbial fuel cells in relation to different carbon-based anode materials. Energy 2016, 106, 277-284. [CrossRef]

22. Catal, T.; Kul, A.; Atalay, V.E. Efficacy of microbial fuel cells for sensing of cocaine metabolites in urinebased wastewater. J. Power Sources 2019, 414, 1-7. [CrossRef]

23. Włodarczyk, P.P.; Włodarczyk, B. Microbial Fuel Cell with Ni-Co Cathode Powered with Yeast Wastewater. Energies 2018, 11, 3194. [CrossRef]

24. Zhou, S.; Lin, M.; Zhuang, Z. Biosynthetic graphene enhanced extracellular electron transfer for high performance anode in microbial fuel cell. Chemosphere 2019, 232, 396-402. [CrossRef] [PubMed]

25. Kang, H.; Jeong, J.; Gupta, P.L. Effects of brush-anode configurations on performance and electrochemistry of microbial fuel cells. Int. J. Hydrogen Energy 2017, 42, 27693-27700. [CrossRef] 
26. Alipanahi, R.; Rahimnejad, M.; Najafpour, G. Improvement of sediment microbial fuel cell performances by design and application of power management systems. Int. J. Hydrogen Energy 2019, 44, 16965-16975. [CrossRef]

27. Ma, D.; Jiang, Z.H.; Lay, C.H. Electricity generation from swine wastewater in microbial fuel cell: Hydraulic reaction time effect. Int. J. Hydrogen Energy 2016, 41, 21820-21826. [CrossRef]

28. López Zavala, M.Á.; González Peña, O.I. Use of Cyclic Voltammetry to Describe the Electrochemical Behavior of a Dual-Chamber Microbial Fuel Cell. Energies 2019, 12, 3532. [CrossRef]

29. Jia, Y.; Feng, H.; Shen, D. High-performance microbial fuel cell anodes obtained from sewage sludge mixed with fly ash. J. Hazard. Mater. 2018, 354, 27-32. [CrossRef]

30. Tremouli, A.; Karydogiannis, I.; Pandis, P.K. Bioelectricity production from fermentable household waste extract using a single chamber microbial fuel cell. Energy Procedia 2019, 161, 2-9. [CrossRef]

31. Guo, J.; Cheng, J.; Li, B. Performance and microbial community in the biocathode of microbial fuel cells under different dissolved oxygen concentrations. J. Electroanal. Chem. 2019, 833, 433-440. [CrossRef]

32. Chen, J.; Zhang, L.; Hu, Y. Bacterial community shift and incurred performance in response to in situ microbial self-assembly graphene and polarity reversion in microbial fuel cell. Bioresour. Technol. 2017, 241, 220-227. [CrossRef] [PubMed]

33. Li, M.; Zhou, M.; Tian, X. Microbial fuel cell (MFC) power performance improvement through enhanced microbial electrogenicity. Biotechnol. Adv. 2018, 36, 1316-1327. [CrossRef] [PubMed]

34. Sekrecka-Belniak, A.; Toczyłowska-Mamińska, R. Fungi-Based Microbial Fuel Cells. Energies 2018, 11, 2827. [CrossRef]

(C) 2020 by the authors. Licensee MDPI, Basel, Switzerland. This article is an open access article distributed under the terms and conditions of the Creative Commons Attribution (CC BY) license (http://creativecommons.org/licenses/by/4.0/). 